\title{
Fixed Drug Eruption: Case Report
}

\author{
$\underline{\text { Azhari Gani }}{ }^{1}$, Muhammad Jailani ${ }^{2}$ \\ ${ }^{1}$ Department of Internal Medicine, Faculty of Medicine, Universitas Syiah Kuala, Banda Aceh, Indonesia \\ ${ }^{2}$ Plastic, Reconstructive and Aesthetic Surgery Division, Faculty of Medicine, Universitas Syiah Kuala, \\ Banda Aceh, Indonesia \\ Email: azharigani@unsyiah.ac.id
}

\begin{abstract}
:
Drug allergy is characterized by hypersensitivity reactions to pharmacological agents, having an immune mechanism of development. In these reactions antibody and/or activated $T$ cells are directed against medications or their metabolites. This problem is rather urgent for practical healthcare, as over $7 \%$ of people suffer from drug allergy. A 54-year old male presented at the emergency room Hospital Zainoel Abidin with redness and swelling of the bodies of a burning sensation, itching pain. In the detailed anamnesis taken from the patient, it was learned that 1 days previously he had consulted a nurse with the fever and 60 min after examination had been prescribed with acid mefenamic. Hypersensitivity immune reactions to medications, according to the present concepts, are divided into immediate reactions (within 1-6 $\mathrm{h}$ after starting the preparation manifesting with various forms-from mild to life-threatening symptoms of anaphylaxia), or delayed reactions (several hours to several days after the offending medication is started, manifesting clinically with exanthemas in the majority of cases). Drug allergy should be prevented.Steps to prevent allergic drug reactions include (1) a careful history to determine host risk factors, (2) avoidance of cross-reactive drugs, (3) use of predictive tests when available, (4) proper and prudent prescribing of drugs (especiallyantibiotics) that are frequently associated with adverse reactions, (5) use of oral drugs when possible, and (6) documentation of drug allergy in the patient's medical record.
\end{abstract}

Keywords: drugs; allergy; reactions; hypersensitivity

\section{Introduction}

Drug allergy is characterized by hypersensitivity reactions to pharmacological agents, having an immune mechanism of development. In these reactions antibody and/or activated $\mathrm{T}$ cells are directed against medications or their metabolites. Based on the National Patient Safety Incident Report (Indonesian Hospital Unity Congress / PERSI September 2007), errors in drug administration in Indonesia rank first $(24.8 \%)$ of the top 10 reported incidents (Ministry of Health of the Republic of Indonesia, in Lubis 2020) ${ }^{21}$. This problem is rather urgent for practical healthcare, as over $7 \%$ of people suffer from drug allergy. Moreover, heavy life-threatening allergic reactions may develop demanding hospitalization and long-term treatment. Immunologic reactions to drugs (reactions of drug hypersensitivity) are considered among unfavorable reactions to drugs, category $\mathrm{B}$, whose mechanism is associated with abnormal response to medications. This distinguishes them from type a reactions, which may be in any patient, and, as a rule, are connected with the main mechanism of drug effect and its dosage ${ }^{1,2}$.

Theoretically allergic reactions may be induced by all medicines, however the most common cause of them are antibiotics, anticonvulsant preparations, nonsteroidal antiinflammatory drugs (NSAID), anesthetics. The problem of drug abuse has very broad and complex dimensions; both from a medical, psychiatric, mental health, as well as psychosocial (economic, political, socio-cultural, criminal, etc.) perspective ${ }^{22}$. The risk of drug allergy, its 
clinical characteristics depend on individual properties of the immune system, drug dose, treatment duration, the route of administration, patient's sex, and also on the unique HLA-signs, which are described in increasing frequency. Immune and nonimmune (pseudoallergic) forms of hypersensitivity reactions can develop to the medicinal preparations, often having identical clinical. Immediate and delayed depending on the time of theirmanifestation after starting the treatment. Immediate drug hypersensitivity reactions develop mainly within an hour (the first six hours) after medication intake and are predominantly induced by $\mathrm{IgE}$-mediated mechanism. Their typical symptoms are urticaria, angioneurotic edema, rhinoconjuctivitis, bronchospasm, nausea, vomiting, diarrhea, pain in the abdomen, anaphylaxia. Delayed allergic reactions may be realized at any time an hour after the drug introduction, but usually occur $6-72$ hour after the medication intake and are connected mainly with a $T$ cell mechanism of allergic reaction. Their clinical manifestations are diverse and may include maculopapular exanthema, exfoliative dermatitis, erythrodermia, DRESS-syndrome (drugrelated eosinophilia with systemic symptoms), toxic epidermal necrolysis, and other bulleos reactions. General systemic effects may comprise development of hepatitis, nephritis, cytopenia ${ }^{1,3}$.

Nonsteroidal anti-inflammatory drugs have been reported tobe the second most common cause of drug-induced hypersensitivity reactions. Aspirin (ASA) hypersensitivity affects from $0.5 \%$ to $1.9 \%$ of the general population. Prevalence of hypersensitivity to NSAIDs among adult asthmaticpatients assessed by questionnaires or medical records isranging from $4.3 \%$ to $11 \%$ and can be as high as $21 \%$ if diagnosis includes provocation tests. Among patients with bronchial asthma and nasal polyps, the prevalence of ASA hypersensitivity may reach $25.6 \%$. Cutaneous manifestations to NSAIDs may affect $0.3 \%$ of general population, and the prevalence of aspirin hypersensitivity may be as high as $27-35 \%$ in patients with chronic urticaria. NSAIDs have been reported to be the major or the second group of drugs responsible for anaphylactic reactions. The prevalence of delayed-type skin or systemic reactionsto NSAIDs is not known, but severe lifethreatening skin reactions as erythema multiforme, toxic epidermal necrolysis(TEN), StevensJohnson syndrome (SJS) or drug reactions with eosinophilia and systemic symptoms (DRESS) are rare. Incidence of TEN in the general population has been estimated at $0.4-1.2$ and for SJS at 1-6 cases per million person-years. Cutaneus skin reactions occur in approximately 2 to 3 percent of patient taking drugs. Fixed drug eruptions are less common than exathematous eruptions, which are estimated to accout for up to 95 percent of cutaneous drug reactions. Fixed drug eruptions account for 14 to 22 percent of cutaneus drug reaction ${ }^{4,5}$.

\section{Review of Literatures}

Hyperproduction of $\mathrm{IgE}$ antibodies by antigenspecificB lymphocytes underlies immediate allergic drug hypersensitivity reactions. Binding of specific $\operatorname{IgE}$ antibodies to highly affinic receptors on the surface of mast cells and basophils, their interaction with the drug antigen results in the release of preformed mediators (histamine, tryptase), tumor necrosis factor and newly formed mediators (leukotriens, prostaglandins, kinins, cytokines). These mediators can be used as diagnostic biomarkers of drug hypersensitivity. Clinically these reactions manifest with urticaria, angioedema, rhinitis, conjunctivitis, bronchspasm, gastrointestinal disorders or anaphylaxia, anaphylactic shock. Their development may be observed in application of foreign sera, beta-lactam antibiotics, sulphanilamides, analgizing agents, NSAIDs ${ }^{1,6}$.

Factors associated with an increased risk of developing a drug allergy include age, gender, genetic polymorphisms, certain viral infections and drug-related factors (e.g., frequency of exposure, route of administration, molecular weight). Drug allergy typically occurs in young and middle-aged adults, and is more common in women than men. Genetic polymorphisms in the 
human leukocyte antigen (HLA; a gene product of the major histocompatibility complex) as well as viral infections such as human immunodeficiency virus (HIV) and the Epstein-Barr virus (EBV), have also been linked to an increased risk of developing immunologic reactions to drugs. Susceptibility to drug allergy is influenced by genetic polymorphisms in drug metabolism. In addition, topical, intramuscular, and intravenous routes of administration are more likely to cause allergic drug reactionsthan oral administration; while intravenous administrationis associated with more severe reactions. Prolonged high doses or frequent doses are more likely to lead to hypersensitivity reactions than a large single dose. Furthermore, large macromolecular drugs (e.g., insulin or horse antisera) or drugs that haptenate (bind to tissue or blood proteins and elicit an immune response), such as penicillin, are also associated with a greater likelihood of causing hypersensitivity reactions. Although atopic patients do not have an increased risk for drug allergy, they are at increased risk for serious allergic reactions ${ }^{7,8}$.

Hypersensitivity to NSAIDs can be classified according to the time of onset and the clinicalmanifestations into acute and delayed. Acute reactions start immediately to several hours after drug administration and include:

(1) Respiratory reactions: Observed in patients with Aspirin Exacerbated Respiratory Disease (AERD), also called aspirin triad, Samter's disease, or aspirin intolerant asthma. These individuals experience a chronic disease characterized by chronic rhinosinusitis, severe persistent and steroid-dependent asthma, with or without nasal polyposis. Acute asthma exacerbations occur when they receive aspirin or classic NSAIDs. These asthma attacks aresevere and may be life-threatening. Various genetic polymorphisms have been associated with this condition.

(2) Cross reacting urticaria and angioedema: Exacerbations of urticaria and/or angioedema induced by COX-1 inhibitors are observed in up to one third of patients with chronic urticaria, more often with drugs of the heteroaryl group (naproxen, diclofenac, ibuprofen). Various genetic polymorphisms, including genes coding for HLA antigens, LTC4 synthase, 5 lipooxygenase, and the high affinity receptor for $\mathrm{IgE}$ have been observed in these patients.

(3) Urticaria, angioedema and anaphylaxis induced by multiple NSAIDs: In patients who do not suffer other morbid conditions NSAIDs can precipitate acute urticaria, angioedema or systemic reactions. This variant of hypersensitivity is more prevalent in atopic individuals and facial angioedema is the most frequent clinical manifestation. It has been associated with A444-C allele of LTC4 synthase.

(4) Urticaria, angioedema and anaphylaxis induced by a single NSAID: More frequently triggered by pyrazolones, but also reported for aspirin, paracetamol, ibuprofen, diclofenac and naproxen. These reactions constitute about 30\% of adverse reactions to NSAIDs and are observed with increased frequency in patients with previous history of atopic disease, food or drug allergy. The clinical manifestations include urticaria, angioedema, laryngeal edema, anaphylaxis, generalized pruritus, rhinitis or bronchospasm ${ }^{4,9}$.

Delayed reactions begin after 24 hours of NSAID exposure, can be induced by a single or multiple cross-reacting NSAIDs, and are clinically expressed either as organ specific or as multisystemic diseases. Examples of organ specific diseases are: 
1) Skin: Maculopapular exanthemas, Fixed drug eruptions, Bullous reactions (erythemamultiforme, Stevens-Johnson syndrome, toxic epidermal necrolysis), acute generalized exanthematous pustulosis, contact and photocontact dermatitis.

2) Lung: Pneumonitis.

3) Central nervous system: Aseptic meningitis.

4) Kidney: Nephritis ${ }^{4,7,10}$.

In recent years special attention has been drawn to delayed allergic reactions to medications, which are mediated by $\mathrm{T}$ lymphocytes. The most common target for $\mathrm{T}$ lymphocytes responding to medications is skin, but other organs may also be involved in the process. First, processing of drug antigen by dendrite cells if performed, and then antigen is transported to the regional lymph nodes, where it is presented by $\mathrm{T}$ cells. Later antigen-specific $\mathrm{T}$ lymocytes migrate to the targeted organ, and after antigen exposure they are activated and secrete proinflammatory cytokines, which cause inflammation and damage of the tissue. Clinically delayed drug hypersensitivity reactions manifest with dermatologic symptoms: itching maculopapulous rash, fixed drug rashes, vasculitis, toxic epidermal necrolysis, and Stevens-Johnson syndrome, generalized bullous fixed drug rashes, acute generalizedexanthematous pustulosis and symmetric drug-relatedintertrigous exanthemas, and located on extensor surfaces of the limbs. Internal organs can also be affected by the pathological process (isolated or in combination with dermatologic symptoms resulting in hepatitis, kidney damage, hypersensitivity pneumonitis, cytopenial $^{11,12}$.

As shown above, clinical manifestations of drug hypersensitivity can be immediate and delayed relative to the time of starting the medication. Additionally, systemic (anaphylaxia, drug fever, serum sickness) and organospecific variants of drug allergic reactions are distinguished. In current publications, skin is considered to be the main target organ in drug hypersensitivity, though other organs can be involved in the pathological process: hemopoiesis system (eosinophilia, cytopenia, hemolytic anemia), respiratory system (rhinitis, bronchospasm, laryngeal edema, pulmonary eosinophilic infiltrate), urinary system (glomerulonephritis, nephritic syndrome, interstitial nephritis), hepatobilliar system (hepatocellular lesions, cholestasis) ${ }^{1,9,13}$.

The four main types of immune reaction process, types $1-3$ are mediated principally by antibodies of different classes while type 4 processes are mediated by $T$ cells but there are many different clinical syndromes that can be produced. The mechanistic aspects will be considered first after which the clinical distinctions will be explained. Type 1 hypersensitivity involves antigen-specific $\mathrm{IgE}$ bound on the surface of mast cells and basophils. Interaction with antigen degranulates the cells with release of mediators including histamine, prostaglandins and leukotrienes. This results in rapid vasodilatation and increased vascular permeability producing redness and oedema in the skin (urticarial and sometimes angioedema), or contraction of smooth muscle resulting in bronchoconstriction (asthma) and/or intestinal cramps and diarrhoea,If the mediator release is very great, the systemic syndrome of anaphylaxis results. Type 2 reactions involve binding of antibodies (usually $\mathrm{Ig} G$ ) to antigenic determinants on the surface of various cell types. In some drug-induced reactions the drug is part of the antigen. in others, such as pemphigus foliaceous auto-antibodies target self-proteins and the precise role of the drug is unclear. Type 3 reactions involve circulating immune complexes in which $\operatorname{IgG}$ antibodies of relative low affinity are complexed with the antigen in small complexes. The complexes adhere to endothelial surfaces and fix complement, which induces accumulation and activation of neutrophils resulting in damage to the endothelium sufficient to allow extravasation of erythrocytes and the clinical syndrome of allergic or hypersensitivity vacuities with purpuric lesions in skin. Type 4 reactions are classically referred to as 'delayed type' because they develop 
over many hours. Investigation of the reactions in skin has led to the realization that $\mathrm{T}$ lymphocytes can deploy a wide range of mediators and effector mechanisms that generate very different clinical manifestations. We emphasise that appreciation of this mechanistic classific ation is principally of importance in consideration of the very similar clinical patterns of weal-like lesions that can be generated by type $1 \mathrm{IgE}$-mediated mechanisms and type 4, T cell-mediated mechanisms. As will be seen below, the diagnostic and prognostic implications can be extremely different $1^{4,13}$.

Fixed dermatitis is an interesting type of drug rash, consisting of one or several elements (erymatous, bullous, in the form of plates), of various shapes and sizes, with distinguished boundaries. They are established to occur in one and the same place each time the preparation is introduced. Discontinuance of the medicine is usually accompanied by reduction of symptoms but often with retained hyperpigmentation, which allows easy determination of the affected area. If the drug is introduced a second time, symptoms recur within $2 \mathrm{~h}$, the number of elements often increases. This clinical variant is usually associated with CD8+ T cells. When the area of skin involvement is not large, the course is likely to be favorable, but in the extensive process with the systemic symptoms in the form of fever and arthralgia the prognosis may be not so optimistic and differential diagnosis includes Stevens-Johnson syndrome1, 15, and 16.

The existing scientific data do not allow formation of comprehensive complex of measures on diagnosing drug allergy. In this connection, methods of general clinical diagnosis continue to be of determining value, especially history-taking (allergologic, pharmacologic and family history), general clinical examination with revealing the main syndromes typical of drug allergy. To diagnose some clinical and pathogenetic variants of drug allergy, in vivo testing and some biological in vitro testing can be performed. However, the list of certified methods of drug allergy investigation available for practical use is rather scanty.

The majority of methods remained within the frames of study projects. In vivo methods (skin tests, provoking tests) are usually economically affordable and clinically informative. However, these tests can be performed only 4-6 weeks after stopping drug hypersensitivity reaction, and require observation of special conditions. This reduces their value as they cannot be applied for emergency diagnosis and therapy (post-factum diagnosis). When it is impossible to exclude the diagnosis of drug allergy on the basis of medical history and clinical data, specific allergological diagnosis should be carried out in specialized centers. It will assistin establishing a diagnosis and recommendingalternative pharmacotherapy. Allergological diagnosis(skin, provocation) can be performed after gatheringallergological and pharmachological history. Allergologicalexamination is often required to confirm allergicnature of drug hypersensitivity reactions relative toantibiotics, NSAIDs, and anesthetics. Skin testing is an available method forhypersensitivity reaction diagnosis. However, information about the development of standarddiagnostic allergens on the basis of medications hasnot been found.

Prick testing andintradermal tests are especially important to identify IgErelatedmechanisms of drug allerg. Prick-tests arerecommended for primary screening examination.Intradermal testing can be performed in case of negativeprick test findings, they are informative enough in caseof immediate hypersensitivity reactions to beta-lactamantibiotics, heparin, and sometimes in delayed reactionsas well. To determine the possibility of T cellmediateddrug hypersensitivity delayed reactions, patch testing (application skin tests) and/or intradermal tests areperformed. In some cases negative results ofskin testing can be explained by the fact, that it is notthe medication but its metabolites possess immunogenicproperties. In such situations drug provoking tests can be used to confirm the diagnosis. Drug provocation testis 
the golden standard for identification of the drug having caused the development of hypersensitivity reactions. Provocation tests with a drug which is supposed to cause the sideeffect can confirm or exclude the diagnosis of drug hypersensitivity reaction. Such tests can be done as early as 1 month after the primary drug allergic reaction by a specially trained personnel in specialized centers having an experience in early identification of hypersensitivity, reactions, and capable of rendering an adequate aid in case of life-threatening conditions. Contraindication to provocation tests is availability of life-threatening drug hypersensitivity reaction (anaphylactic shock, other systemic reactions such as Stevens-Johnson syndrome, toxic epidermal necrolysis, vasculitis). The route of introducing the suspected medication in provocation tests is mainly the same as in its initial application. But preference is given to peroral route, as it is connected with a lower risk of drug hypersensitivity reaction development. Patch testing is an essential investigation in patients with persistent eczematous eruptions when contact allergy is suspected or cannot be ruled out. Patch testing may help to identify allergens that should be avoided and is discussed separately. The thin-layer rapid-use epicutaneus (TRUE) test, which include 35 allergen and one control, is a commercially available ready-to-use test widely used in basuc stabdar patch testing among dermatologists and allergists ${ }^{8,17,18}$.

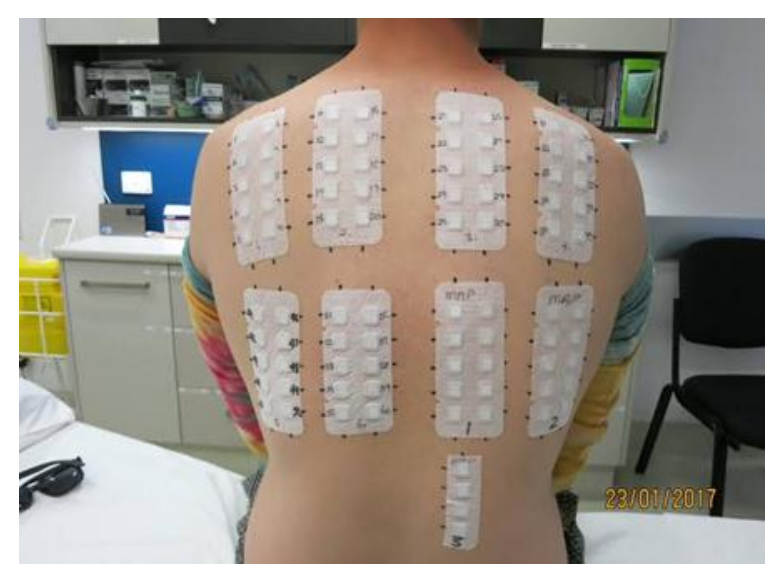

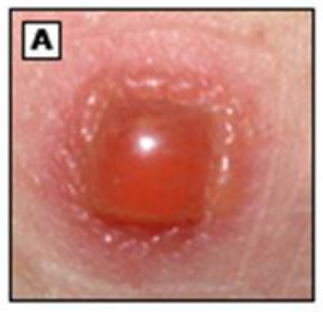

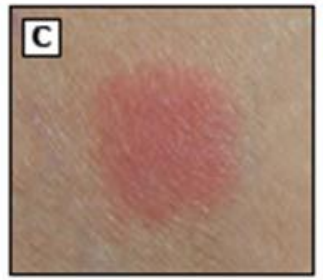

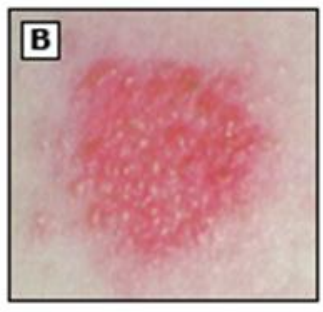

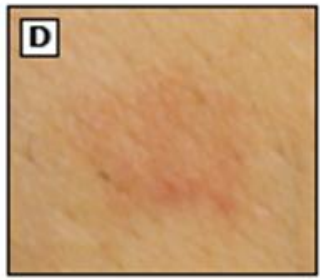

Figure 1. Patch test. (A) +++ reaction. (B) ++ reaction (note the microvesicles). (C) + reaction. (D) + - reaction 18

The development ofbiological methods of diagnosing drug hypersensitivity reactions is believed to be a very promising direction. Such methods are advantageous for the patients receiving multi-drug therapy and in heavy hypersensitivity reactions, when in vivo tests with medications are contraindicated. This kind of examinations is safe for the patient and is possible to be performed at the peak of clinical manifestations. Among in vitro tests the majority of methods implemented into clinical practice are based on measurement of allergen-specific IgE antibodies to drug allergens. However, drug hypersensitivity IgE-related reactions seem to be less common than delayed hypersensitivity reactions (T lymphocytes-mediated). Besides, commercial kits for identifying specific IgE are available for a limited number of medicationsincluding amoxicillin, ampicillin, cephaclor, penicillin, insulin (bovine, porcine, human), adrenocorticotropic hormone, suxamethonium and some other preparations ${ }^{8}$. 


\section{Case Report}

A 54-year old male presented at the emergency room Hospital Zainoel Abidin with redness and swelling of the bodies of a burning sensation, itching pain. In the detailed anamnesis taken from the patient, it was learned that 1 days previously he had consulted a nurse with thefever and 60 min after examination had been prescribed with acid mefenamic. The patient stated that he had 7 timespreviously same experienced any allergic reaction to anymedication such as amoxicillin, metampiron and acid mefenamicover the last 10 years, nobody in family has any allergic to any medication. In the vital sign, patient compos mentis, blood pressure : 130/80 mmhg, heart rate 110 times per minutes, respiratory rate $38,5^{\circ} \mathrm{C}$. In the body examination,eyes, thorax, abdomen are normally, there was seen to be anepithelial rash on the skin around the redness, with well demarcated, round to oval, dusky red and brown/black macules and plaques with overlying large flaccid bullae. In the laboratory : haemoglobin $16 \mathrm{~g} / \mathrm{dl}$, leukocyte $8.200 / \mathrm{mm} 3$, thrombocyte $140.000 / \mathrm{mm} 3$, diftell count $0 / 0 / 1 / 86 / 8 / 5$.

Patient were diagnosed fixed drug eruption, inpatient 4 days, he has treated diet 1700 $\mathrm{kkal} /$ days, Ringer Lactat Infussion 30 macro drop per minute, intravenous methyl prednisolone $125 \mathrm{mg} / 12 \mathrm{~h}$, intrevenous dipenhidramin $10 \mathrm{mg} / 8 \mathrm{~h}$, intravenous omeprazole $40 \mathrm{mg} / 12 \mathrm{~h}$. He has controlled at polyclinik alergy internal medicine with methyl prednisolone $20-20-16 \mathrm{mg}$, ciprofloxacin $500 \mathrm{mg}$ twice a day, cetirizin $10 \mathrm{mg}$ twice a day, omeprazole $20 \mathrm{mg}$ twice a day, inerson oint and thiamycin $2 \%$ zalf topical. Patient has educated thathe must avoidance of cross reactive drugs such us antibiotic penisillin and NSAID.At thefollow-up examination, the rash and the redness and swelling in the body were have recovered.

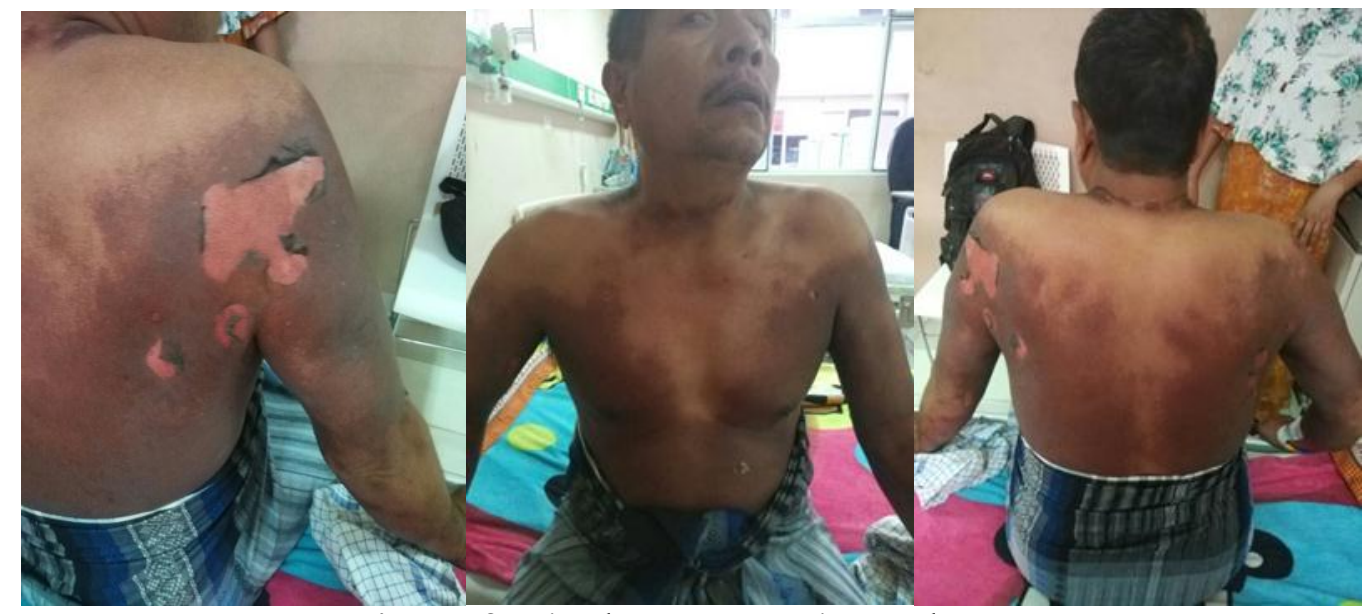

Figure 2. Fixed Drug Eruption 2nd Days

\section{Discussion}

Hypersensitivity immune reactions to medications, according to the present concepts, are divided into immediate reactions (within 1-6 h after starting the preparation manifesting with various forms - from mild to life-threatening symptoms of anaphylaxia), or delayed reactions (several hours to several days after the offending medication is started, manifesting clinically with exanthemas in the majority of cases). Dermatologicsymptoms are the most frequent in drug allergy, duecto a high immune activity of the skin. Rashes are of polymorphic character. They are accompanied by itching, which is most intensive in measles-like or scarlatiniform rash. Papulous and/or measleslike rash compose $75-90 \%$ of drug-induced skin eruptions ${ }^{1,14}$. 
Dermatologic symptoms are the most frequent in drug allergy, due to a high immune activity of the skin. Rashes are of polymorphic character. They are accompa nied by itching, which is most intensive in measles-like or scarlatiniform rash. Papulous and/or measleslikerash compose $75-90 \%$ of drug-induced skin eruptions. The onset of rash is observed, as a rule, 1 week after medication exposure. They are not usually dangerous, if there are no other manifestations. Cytotoxic CD4+ T cells are the prevailing type of cells in this case. However, progression of eruptions to more serious manifestations, including toxic epidermal necrolisis, which is mainly mediated by CD8+ cytotoxic T cells, is possible. These cutaneous changes mostly disappear some days after the preparation is discontinued, which is often accompanied by peeling of epidermis, leaving areas of discoloration. The main difficulty of clinical diagnosis of these pathological conditions is to differentiate them from infectious exanthems. Some clinical variants of drug hypersensitivity are realized in a certain combination of infectious agents and medications. An example is the risk of exanthema occurrence in using antibacterialpreparations of aminopenicillin group in patients with infection, caused by Epstein-Barr virus ${ }^{1,19,20}$.

The terminology proposed by the Nomenclature Committee of the World Allergy Organization that defines drug hypersensitivity as the symptoms or signs initiated by exposure to a drug at a dose normally tolerated by non-hypersensitive persons. "Drug allergy" refers to immunologically mediated drug hypersensitivity reactions. These may be either immunoglobulin $\mathrm{E}(\mathrm{IgE}$ )-mediated (immediate) or non IgE-mediated (delayed). "Non allergic hypersensitivity reactions" refer to adverse drug reactions that are not mediated by immunological mechanisms. Cutaneous skin reactions occur in approximately 2 to 3 percent of patients taking drugs. FDEs are less common than exanthematous (morbilliform) eruptions, which are estimated to account for up to 95 percent of cutaneous drug reactions. FDEs occur in both sexes and in all age groups; in children, FDEs account for 14 to 22 percent of cutaneous drug reactions ${ }^{4,5}$.

Since NSAID hypersensitivity has multiple clinical manifestations, the mechanisms incriminated ineach of them are different. Reactions to aspirin and NSAIDs observed in patients with AERD are mediated by inhibition of COX-1, leading to a shunting ofarachidonic acid metabolism towards the 5-lipoxygenase pathway and increased production of cysteinyl leukotrienes. A decreased productionof PGE2, a modulator of mediator release from mast cells and other inflammatory cells, also plays a role ${ }^{4}$.

Acetylsalicylic acid (ASA) and NSAIDs can cause bothtrue allergic and pseudoallergic reactions, includingexacerbations of underlying respiratory diseases, urticaria,angioedema, and anaphylaxis. Patients with underlyingchronic respiratory diseases, such as asthma, rhini tis and sinusitis, may react to ASA and NSAIDs that inhibit cyclooxygenase-1 (COX-1). Patients with AERD must avoid all COX-1 inhibitors, including aspirin, in order to avoid the occurrence of serious asthma exacerbations. For the treatment of pain and inflammation NSAIDs that do not inhibit COX-1, such as acetaminophen in doses below 1,000 mg and COX-2 inhibitors are recommended after challenge in the office or medical facility. Aspirin desensitization is indicated for patients who require continuous anti-inflammatory or anti-thrombotic therapy, such as those with ischemic heart disease or chronic arthritis ${ }^{4,8}$.

FDE typically presents with well-demarcated, round to oval, dusky red to brown/black macules that may evolve into edematous plaques with or without vesiculation or blistering. Lesions are usually solitary, buy may occur in small groups. Systemic symptoms, such as fever and malaise, are usually absent. Pruritus and a burning or stinging sensation are common. However, the estimated frequency of these findings is not known because they are not 
consistently reported in case series. FDE may occur anywhere on the body. Sites of predilection include the lips, genitalia, perianal area, hands, and feet. On mucosal areas, erosions and ulcers may develop. FDE occasionally develops at the site of an antecedent trauma (eg, insect bite, burn, venipuncture). Acute lesions generally appear 30 minutes to 8 hours after drug administration, but can occur up to two weeks after drug exposure. After discontinuation of the offending drug, lesions resolve spontaneously in 7 to 10 days, leaving a persistent gray/brown or slate gray postinflammatory hyperpigmentation. Upon reexposure to the offending drug, lesions typically recur in the same site, but new lesions may develop elsewhere. After one or more localized eruptions, FDE rarely may evolve into a bullous generalized form mimicking Stevens Johnson syndrome/toxic epidermal necrolysis ${ }^{5}$.

A 54-year old male presented at the emergency room Hospital Zainoel Abidin with redness and swelling of the bodies of a burning sensation, itching pain. In the detailed anamnesis taken from the patient, it was learned that 1 days previously he had consulted a nurse with the fever and 60 min after examination had been prescribed with acid mefenamic. The patient stated that he had 7 times previously same experienced any allergic reaction to anymedication such as amoxicillin, metampiron and acid mefenamic over the last 10 years, nobody in family has any allergic to any medication. In the vital sign, patient compos mentis, blood pressure : 130/80 mmhg, heart rate 110 times per minutes, respiratory rate 38,50 C. In the body examination, eyes, thorax, abdomen are normally, there was seen to be anepithelial rash on the skinaround the redness, with well demarcated, round to oval, dusky red and brown/black macules and plaques with overlying large flaccid bullae around the redness and swelling of the bodies. In the laboratory : haemoglobin $16 \mathrm{~g} / \mathrm{dl}$, leukocyte $8.200 / \mathrm{mm} 3$, thrombocyte $140.000 / \mathrm{mm} 3$, diftell count $0 / 0 / 1 / 86 / 8 / 5$.

The treatment of FDE is largely symptomatic and aimed at the relief of pruritus. The efficacy of symptomatic therapies for the treatment of FDE has not been evaluated in randomized trials. However, their use is based on clinical experience and indirect evidence of benefit in patients other pruritic skin conditions. For patients with single or a small number of lesions, we suggest medium to high potency topical corticosteroids and systemic antihistamines. Topical corticosteroids are applied two times per day for 7 to 10 days. Oral H1 antihistamines are generally used, including:

- Diphenhydramine25 to $50 \mathrm{mg}$ orally every four to six hours for adults and children $\geq 12$ years; 12.5 to $25 \mathrm{mg}$ orally every four to six hours for children 6 to 11 years; and $6.25 \mathrm{mg}$ orally every four to six hours for children 2 to 5 years. Diphenhydramine is continued until pruritus subsides.

- Hydroxyzine $25 \mathrm{mg}$ orally three to four times per day for adults and children $\geq 6$ years; 2 $\mathrm{mg} / \mathrm{kg}$ per day orally divided every six to eight hours for children $<6$ years. Hydroxyzine is continued until pruritus subsides.

For patients with generalized FDE or generalized bullous FDE, particularly if systemic symptoms are present, a short course of moderate dose systemic corticosteroids (eg, prednisone 0.5 to $1 \mathrm{mg} / \mathrm{kg}$ per day for three to five days) may be beneficial ${ }^{5}$.

Patient were diagnosed fixed drug eruption inpatient 4 days, he has treated diet 1700 $\mathrm{kkal} /$ days, Ringer Lactat Infussion 30 macro drop per minute, intravenous methyl pred nisolone $125 \mathrm{mg} / 12 \mathrm{~h}$, intrevenous dipenhidramin $10 \mathrm{mg} / 8 \mathrm{~h}$, intravenous omeprazole $40 \mathrm{mg} / 12 \mathrm{~h}$. He has controlled at polyclinik alergy internal medicine with methyl prednisolone $20-20-16 \mathrm{mg}$, ciprofloxacin $500 \mathrm{mg}$ twice a day, cetirizin $10 \mathrm{mg}$ twice a day, omeprazole $20 \mathrm{mg}$ twice a day, 
inerson oint and thiamycin $2 \%$ zalf topical. Patient has educated that he must avoidance of cross reactive drugs such us antibiotic penisillin and NSAID. At thefollow-up examination, the rash and the redness and swelling in the body were have recovered.

Ideally drug allergy should be prevented. Steps to prevent allergic drug reactions include (1) a careful history to determine host risk factors, (2) avoidance of cross-reactive drugs, (3) use of predictive tests when available, (4) proper and prudent prescribing of drugs (especially antibiotics) that are frequently associated with adverse reactions, (5) use of oral drugs when possible, and (6) documentation of drug allergy in the patient's medical record ${ }^{5}$.

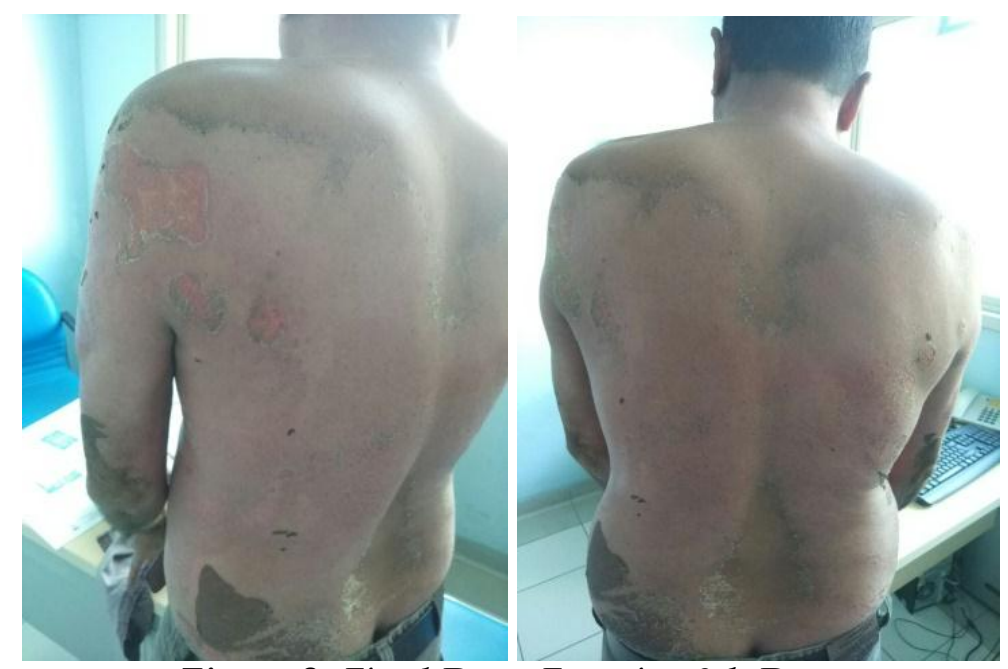

Figure 3. Fixed Drug Eruption 9th Days

\section{Conclusion}

Hypersensitivity immune reactions to medications, according to the present concepts, are divided intoimmediate reactions (within $1-6 \mathrm{~h}$ after starting thepreparation manifesting with various forms - frommild to life-threatening symptoms of anaphylaxia),or delayed reactions (several hours to several daysafter the offending medication is started, manifesting clinically with exanthemas in the majority of cases). Specific diagnosis of drug allergy is performed using in vivo tests (prick tests, intradermal tests, patch tests, provocation tests) and in vitro test (identification of drug specific IgE test, basophil activation tests, leukocyteblast-transformation reactions, quantitative identification of cytokines and other proteins, e.g. granzyme andtryptase in the peripheral blood). At present not all these methods are accessible in real clinical practice, the list of commercial kits for drug allergy diagnosis is limited. It is especially important in patient managing to rely on history-taking and general clinicalexamination data, to consider the available informationon association of drug allergy.

Drug allergy should be prevented.Steps to prevent allergic drug reactions include (1) a careful history to determine host risk factors, (2) avoidance of cross-reactive drugs, (3) use of predictive tests when available, (4) proper and prudent prescribing of drugs (especiallyantibiotics) that are frequently associated with adverse reactions, (5) use of oral drugs when possible, and (6) documentation of drug allergy in the patient's medical record . 


\section{References}

1. Balabolkin, I, Researcher, C, Novgorod, N, Federation, R. \& Federation, R. Drug Allergic Reactions: Current Views ( review ). CTM J.8, 159-170 (2016).

2. Annick Barbaud. Guidelines for performing skin tests with drugs in the investigation of cutaneous adverse. 45, 321-328 (2001).

3. Ardern-jones, M. R. \& Friedmann, P. S. Skin manifestations of drug allergy. Br. J. Clin. Pharmacol.71, 672-683 (2011).

4. Kowalski, M. L. et al. Hypersensitivity to nonsteroidal anti-inflammatory drugs ( NSAIDs ) classification, diagnosis and management : review of the EAACI / ENDA and GAZLEN / HANNA. 66, 818-829 (2011).

5. Tetsuo Shiohara, MD, P. Fixed drug eruption : a disease mediated by self-inflicted responses of intraepidermal T cells. Eur J Dermatol, 534-537 (2017).

6. Review, G. Multiple-Drug Intolerance Syndrome. 29, (2016).

7. Warrington, R. \& Silviu-dan, F. Immunology Drug allergy. 7, 1-8 (2011).

8. Doctors, E. \& Referral, P. Drug Allergy : The Facts. 1-8 (2017).

9. Management of the Multiple Drug Allergy Syndrome - Case Report. 2004 (2004).

10.Khobragade, Y. Adverse drug reactions to ibuprofen : a case report. (2016). doi:10.18203/2319-2003.ijbcp20160131.

11.Visacri, B. et al. Adverse Drug Reactions and quality deviations monitored by spontaneous reports. 130-137 (2015). doi:10.1016/j.jsps.2014.06.008

12.Patro, N., Panda, M., Jena, M. \& Mishra, S. Multifocal fixed drug eruptions: A case series. Int. J. Pharm. Sci. Rev. Res.23, 63-66 (2013).

13.Newby, M. D. A Case Series On Fixed Drug Eruptions. 2-5 (2014).

14.Ardern-jones, M. R. \& Friedmann, P. S. Skin manifestations of drug allergy. (2011). doi:10.1111/j.1365-2125.2010.03703.x

15.Weiss, M. E. et al. Drug Allergy : An Updated Practice Parameter. ANAI105, 259-273.e78 16.Haahtela, T. \& Hannuksela, M. Finnish Allergy Programme 2008 - 2018 - time to act and change the course. 634-645 (2018). doi:10.1111/j.1398-9995.2008.01712.x

17.Report, C. Case Report Tramadol Induced Fixed Drug Eruptions - A Case Report. 2, 4-5 (2013).

18.Brod, B. A, Guideline for the management of contact dermatitis, 5, 946 (2009).

19.Titchen, T., Cranswick, N. \& Beggs, S. Adverse drug reactions to nonsteroidal antiinflammatory drugs, COX-2 inhibitors and paracetamol in a pediatric hospital. 1-6 
(2005). doi:10.1111/j.1365-2125.2005.02444.x

20. Journal, W. \& Pharmaceutical, O. F. Paracetamol Allergy : A Case Report. 3, 23-26 (2017).

21. Lubis, F. I., Zulfendri, and Jumirah. (2020). Collaboration the Role of Doctors and

Pharmacers in Rational Preparation in Rantauprapat Hospital, Constraints and Its Efforts.

Britain International of Exact Sciences (BIoEx) Journal Vol. 2 (1):19-28.

22.Zulkarnain. (2019). Eradication Efforts of Drug Abuse in Indonesia. Britain International of Humanties and Social Sciences(BIoHS)Journal Vol. 1 (1): 26-34. 